\title{
The effect of emotion on interpretation and logic in a conditional reasoning task
}

\author{
ISABELLE BLANCHETTE \\ University of Manchester, Manchester, England
}

\begin{abstract}
The effect of emotional content on logical reasoning is explored in three experiments. The participants completed a conditional reasoning task (If $p$, then $q$ ) with emotional and neutral contents. In Experiment 1, existing emotional and neutral words were used. The emotional value of initially neutral words was experimentally manipulated in Experiments $1 \mathrm{~B}$ and 2, using classical conditioning. In all experiments, participants were less likely to provide normatively correct answers when reasoning about emotional stimuli, compared with neutral stimuli. This was true for both negative (Experiments 1B \& 2) and positive contents (Experiment 2). The participants' interpretations of the conditional statements were also measured (perceived sufficiency, necessity, causality, and plausibility). The results showed the expected relationship between interpretation and reasoning. However, emotion did not affect interpretation. Emotional and neutral conditional statements were interpreted similarly. The results are discussed in light of current models of emotion and reasoning.
\end{abstract}

Debates about rationality are prevalent and central in both the emotion and the reasoning literatures (Barrett \& Salovey, 2002; Chater \& Oaksford, 2001; Damasio, 1998; Dawes, 2001; de Sousa, 1987; Ekman \& Davidson, 1994; Evans, Over, \& Manktelow, 1993; Oaksford \& Chater, 1998; Parrott, 1995). Surprisingly, little empirical research has been dedicated to examining the effect of emotion on logicality, which could make an important contribution to the debate. Deductive reasoning in general, and conditional reasoning in particular, are simultaneously prevalent in everyday thinking and representative of the human potential for logical thinking. The three experiments I describe in this article were conducted to explore the effect of emotion on this type of reasoning. The main goal of this research was to compare participants' behavior when reasoning about neutral and emotional contents, and to explore the role of interpretation in this process.

Deductive reasoning is often held as a prime example of human intellectual ability, and the perfect opportunity to look for, or display, logicality. Deductive reasoning is a closed system in which conclusions are derived from premises. Propositions can be judged as true or not based on the prescriptions of normative logic. Tasks such as syllogistic reasoning (e.g., All men are mortal, Socrates is a man, therefore, Socrates is mortal) and conditional reasoning (e.g., If it rains, I get wet. It is raining, therefore, I am getting wet) have been used to explore the ways in

This research was made possible by the support of the British Academy, Grant SG-35169. I thank Sylvain Sirois for reading different drafts of this article. Correspondence concerning this article should be addressed to I. Blanchette, School of Psychological Sciences, University of Manchester, Oxford Road, Manchester M13 9PL, England (e-mail: isabelle.blanchette@manchester.ac.uk). which people reason, and whether this conforms to the prescriptions of logic. Through empirical research, it has become clear that human performance is affected by a number of factors, related to the content or context of the rules, that are not, strictly speaking, relevant within a logical system (Cheng \& Holyoak, 1985; Evans, 1989, 1998; Goel \& Dolan, 2003; Manktelow, 1999; Markovits, 1986; Markovits \& Nantel, 1989; Ohm \& Thompson, 2004).

Whereas there has been much research on the effect of different semantic, informational, or cognitive content variables on reasoning, little research has explored the impact of affective variables. The deductive reasoning paradigm represents an appropriate test bed to empirically explore the widespread, but seldom tested, idea that emotions impair logical reasoning (de Sousa, 1987; French \& Wettstein, 1998; Lyons, 1993). The limited amount of research that does exist on this topic has focused on the effect of psychopathologies, mood, and emotional content on different forms of reasoning.

\section{Emotion and Reasoning: Empirical Findings}

Some studies have investigated deductive reasoning in mood disorders, or psychopathologies with strong emotional components. For instance, it has been shown that depressed patients perform more poorly than healthy controls on syllogistic reasoning tasks (Channon \& Baker, 1994; Radenhausen \& Anker, 1988). This is true both under conditions of exacerbated mood (using a negative moodinduction procedure) and baseline mood conditions.

Other studies have examined reasoning in anxietyrelated disorders and identified distinctive patterns of reasoning related to threat. For instance, in one series of studies, de Jong and colleagues used the Wason selection task to examine participants' reasoning about danger and safety rules (de Jong, Haenen, Schmidt, \& Mayer, 1998; 
de Jong, Mayer, \& van den Hout, 1997; Smeets, de Jong, $\&$ Mayer, 2000). The Wason selection task involves a conditional rule (If $p$ on one side, then $q$ on the other side) and four cards, of which only one side is visible ( $p$, not $p$, $q$, not $q$ ). Participants must select cards to turn over to determine whether the rule is true or not. The authors compared the performance of healthy controls with that of hypochondriacal or phobic patients. They found that participants adopted confirmatory strategies in the case of danger rules (i.e., for If $p$, then $q$ [danger]; participants selected $p$ and $q$ ), but falsificationist strategies in the case of safety rules (i.e., for If $p$, then $q$ [no danger]; participants selected $p$ and not $q$ ). This pattern was present in all participants, but it was magnified in patients reasoning about phobia-specific stimuli.

Thus, research has shown that psychopathologies with important affective components do tend to have an effect on reasoning. However, psychopathologies are complex entities with both emotional and cognitive components. It is difficult to tease out the relative contributions of affective and cognitive factors.

Some research has been conducted with nonclinical samples, looking at the influence of mood on deductive reasoning. Oaksford and colleagues (Oaksford, Morris, Grainger, \& Williams, 1996) induced positive and negative moods in different groups of participants and compared their selections on the Wason task to those of a neutral mood control group. The participants in both positive and negative moods were less likely to provide the normatively correct answers, compared with the control group. Melton (1995) found analogous effects of positive mood on a syllogistic reasoning task. Again, participants in a positive mood were less likely to provide logically valid answers, compared with the control group. Thus, there is evidence that mood, whether positive or negative, may impair logicality on deductive reasoning tasks.

In another recent experiment, the effect of the emotionality of contents (as opposed to emotional state, or mood) was examined (Blanchette \& Richards, 2004). In a conditional reasoning task, the participants were more likely to endorse logical fallacies when reasoning about emotional content (e.g., If someone is in a tragic situation, then she cries), compared with neutral content (e.g., If someone is an actor, then he is an extrovert). One early study by Lefford (1946) provided similar findings. Lefford compared participants' performance on syllogisms with identical logical structures, but different emotional value. The participants were more likely to provide valid answers when reasoning about neutral syllogisms, compared with emotional syllogisms.

Thus, some research suggests that emotional content, as well as mood, may affect reasoning. However, there are intricate confounds in this type of research that are difficult to avoid. The affective and cognitive dimensions of the stimuli are inevitably confounded. Take, for instance, the words terror and desk. The two obviously vary in affective value. However, they also vary on a number of other features. The word terror may be less frequently encountered than the word desk. Salience or rarity might change the allocation of resources in the reasoning task. Semantic associations in long-term memory may also be different in quantity and in kind for the two types of words. There are a number of features that, independently of emotion, may account for the differences found in reasoning about emotional and neutral stimuli. The problem is that to vary the emotionality of the reasoning materials, different semantic vehicles have to be used. The difficulty is exacerbated when entirely different statements are used in the emotional and neutral conditions - for instance, when comparing danger and safety rules (de Jong et al., 1998; de Jong et al., 1997). This problem of isolating affective from cognitive or semantic influences also applies to studies using mood manipulations. Although a wide range of mood-induction procedures exist, many have an important cognitive component, in which different concepts might be primed.

In one study (Blanchette \& Richards, 2004, Experiment 2), we manipulated affective value independently of semantic content, in an attempt to single out the impact of affect on reasoning. We used classical conditioning to manipulate the emotionality of initially neutral words. These words were repeatedly paired with images of either negative, positive, or neutral value. The conditioned words were then used in the reasoning task. The critical feature was that the pairing of words and image type was different across participants, such that the same words were conditioned to become positive for some participants, negative for others, and neutral for yet another group of participants. In this way, we could compare the participants' reasoning about the same semantic contents, but with different emotional content. Using this technique, we also observed a significant effect of emotion on reasoning. The participants were once again less likely to provide normatively correct answers when reasoning about conditioned emotional materials, compared with neutral materials.

Some features of previous experiments (specifically, Blanchette \& Richards, 2004) were important motivations for the present studies. These results showed an effect of emotion on conditional reasoning, using an argument task. In this task, conclusions are presented as questions. For instance, based on the rule If someone is in a tragic situation, then she cries, the participants had to answer a question of the type: Anne is not in a tragic situation. Does she cry? The format of the question could have increased the likelihood that participants would rely on general knowledge, averting the use of formal reasoning. This may have affected the extent to which emotion had an effect on behavior. It is possible that emotion affects reasoning, but not logical reasoning. A more stringent test of the effect of emotion on logicality could be performed. In the experiments reported in this article, a logical verification task was used. The participants were presented with premises and conclusions as statements, and had to determine whether the conclusion logically followed from the premises (e.g., If someone is in a tragic situation, she cries. Anne is not in a tragic situation. She does not cry. Does this conclusion logically follow from the rule?). This type of logical verification task provides a more severe test of the effect of 
emotion on logicality, as opposed to the effect of emotion on reasoning more generally (logical or other).

As in previous studies, conditional reasoning was used. Conditional reasoning involves making inferences on the basis of statements of the type If $p$, then $q$. Although it is formalized in propositional logic, this type of reasoning is prevalent in everyday situations. Based on a conditional statement such as If a person is tired, then she goes to bed, different inferences can be drawn, some of which are logically valid, and some of which are not. Four possible inferences are modus ponens (MP) ( $p$, therefore $q$; Sue is tired, therefore she goes to bed), modus tollens (MT) (not $q$, therefore not $p$; Pat is not going to bed, therefore she is not tired), denying the antecedent (DA) (not $p$, therefore not q; Jade is not tired, therefore she is not going to bed), and affirming the consequent $(\mathrm{AC})(q$, therefore $p$; Liz is going to bed, therefore she is tired). Assuming the rule to be true at all times, the first two inferences (MP and MT) are logically valid but not the latter two (DA and AC).

\section{Emotion, Reasoning, and Interpretation}

The experiments reported in this article also explore a possible mechanism for the effect of emotion on reasoning: the role of interpretation. It is generally accepted that there are at least two processes involved when people reason about conditional statements. Participants must interpret the statements. They must then perform inferences based on the representation produced through this interpretation. The interpretive and inferential mechanisms do not necessarily occur in a strict sequence, but at least some interpretation is required before any inferences can be drawn. According to one view, content and context effects on reasoning are attributable to interpretive factors. In this view, inferential mechanisms may conform to prescriptions of normative logic, but interpretive mechanisms introduce influences that ultimately affect logicality (Henle, 1962; Klaczynski \& Narasimham, 1998; Ohm \& Thompson, 2004; Thompson, 1994; Verbrugge, Dieussaert, Schaeken, \& Van Belle, 2004; Verschueren, Schroyens, Schaeken, \& d'Ydewalle, 2001). According to this hypothesis, if you consider the rule participants are actually reasoning about (i.e., their interpretation of the rule provided by the experimenter), then responses conform to the standards of logic. In other words, content effects occur because participants reinterpret the conditional statements with the influence of their background knowledge. The effect of emotion on reasoning may occur through interpretation, rather than through an influence on inferential components of the reasoning process. For instance, emotional statements may be more likely to be reinterpreted as biconditionals (a possible explanation for the higher rate of endorsement of AC and DA). This hypothesis is tested in the different experiments reported in this article.

To summarize, this article explores the effect of emotional content (emotionality) on logicality in three experiments, using a conditional reasoning task. Two methods for manipulating the emotional connotation of the materials are used. Experiment $1 \mathrm{~A}$ uses existing materials that vary on emotionality. In Experiments $1 \mathrm{~B}$ and 2, emotionality is experimentally manipulated using classical conditioning. The use of this technique makes it possible to disentangle the effect of affective factors from semantic confounds. The reasoning task was designed in such a way as to unambiguously promote logical reasoning. Finally, the contribution of different interpretive variables is measured: perceived sufficiency and necessity (Experiments $1 \mathrm{~A}$ and $1 \mathrm{~B})$, and perceived causality and plausibility (Experiment 2).

\section{EXPERIMENT 1A}

In Experiment 1A, participants reasoned about neutral and emotional conditional statements. Their interpretation of the statements was measured-specifically, perceived necessity and sufficiency. Sufficiency is defined as the degree to which the occurrence of $p$ guarantees the occurrence of $q$. In other words, is $p$ enough to guarantee that $q$ will occur? By contrast, necessity can be defined as the degree to which the absence of $p$ guarantees the absence of $q$. In other words, if $p$ is not present, does that guarantee that $q$ is not present either? Strictly speaking, conditional forms such as If $p$, then $q$ imply a sufficient relation between $p$ and $q$, but not a necessary one. This is termed material implication. Simply put, a rule such as If $p$, then $q$ guarantees that if $p$ occurs, $q$ will also occur, but does not say anything about what will happen if $p$ does not occur.

Although logically speaking, If $p$, then $q$ rules imply sufficiency, but not necessity, statements can be underspecified from a psychological point of view. An increasing amount of research shows that perceived necessity and sufficiency are crucial determinants of participants' reasoning behavior in different conditional reasoning tasks (Ahn \& Graham, 1999; Cummins, Lubart, Alksnis, \& Rist, 1991; Fairley, Manktelow, \& Over, 1999; Liu, 2003; Thompson, 1994, 1995, 2000; Thompson \& Mann, 1995). For instance, when participants interpret a rule as sufficient but not necessary, they are much more likely to provide normatively correct responses (Ahn \& Graham, 1999; Cummins et al., 1991; Fairley et al., 1999). Experiments 1A and 1B explore whether emotionality affects perceived necessity and sufficiency of conditional statements.

\section{Method}

Participants. The participants were students and staff at the University of Manchester. Thirty-two individuals participated in the study in exchange for monetary compensation. There were 19 women and 13 men. The average age was $27(S D=9.9)$, and ranged from 18 to 57 . The first language of most of the participants was English $(n=28)$.

Materials. There were 10 conditional statements of the form If $p$, then $q$. All had the same logical structure. There were five emotional and five neutral rules (see Table 1). The statements were designed in pairs so that they would be as superficially similar as possible. For instance, for four pairs of statements, the antecedents were different (more emotional in one case than the other), but the consequents were identical. These statements were selected from an initial set of 32 that were submitted to a separate group of participants for affective rating. These participants $(n=9)$ rated the emotionality 
Table 1

Reasoning Stimuli Used in Experiment 1A

\begin{tabular}{ll}
\hline \multicolumn{1}{c}{ Neutral } & \multicolumn{1}{c}{ Emotional } \\
\hline $\begin{array}{l}\text { If a child eats too much sugar, } \\
\text { then he has behavioral difficulties }\end{array}$ & $\begin{array}{l}\text { If a child has been sexually abused, } \\
\text { then he has behavioral difficulties } \\
\text { If people drink too much coffee, } \\
\text { then they have trouble sleeping }\end{array}$ \\
$\begin{array}{ll}\text { If you want to catch a bus, } \\
\text { then they have trouble sleeping } \\
\text { then you run }\end{array}$ & $\begin{array}{l}\text { If you see a fire, } \\
\text { then you run }\end{array}$ \\
$\begin{array}{ll}\text { If your mother is looking for you, } \\
\text { then you hide }\end{array}$ & $\begin{array}{l}\text { If you hear a burglar in your house, } \\
\text { then you hide }\end{array}$ \\
$\begin{array}{l}\text { If your boyfriend has been playing football, } \\
\text { then you find dirt on his clothes }\end{array}$ & $\begin{array}{l}\text { If your boyfriend is having an affair, } \\
\text { then you find lipstick on his shirt collar }\end{array}$ \\
\hline
\end{tabular}

of the 32 statements on a scale from 1 (entirely neutral) to 7 (very emotional). Statements were selected to simultaneously maximize superficial similarity between the conditions, and increase differentiation on the emotionality ratings. On average, the emotional statements obtained a rating of $5.04(S D=1.29)$ and the neutral statements $2.63(S D=1.19)[t(8)=4.7, p<.001]$.

For each statement, four associated inferences were presented to participants: modus ponens (MP), modus tollens (MT), affirming the consequent (AC), and denying the antecedent (DA). Specific inferences were constructed by personalizing each of the statements, for instance, Richard has behavioral difficulties, therefore he has been sexually abused (AC).

Procedure. The participants were tested individually and the experiment was run on a computer. For the first part of the experiment, participants were told that they would reason about logical statements of the form If $p$, then $q$, and they were given examples of rules and inferences. They were told that their task was to determine whether the conclusions presented could be logically inferred from the rule. They were explicitly told that some conclusions would be logically valid, whereas others would not. They were also instructed to provide answers based on what logically followed, regardless of whether the conclusion was in fact true or not.

On each trial, the conditional statement was first presented on the screen and stayed on for $3 \mathrm{sec}$. Then, one of the four possible inferences appeared below the statement. The statement and conclusions stayed on the screen until the participants made a response. There was also a reminder of the instructions at the bottom of the screen, in gray, prompting the participants to Press Yes if the conclusion follows logically from the rule, and press No if it does not follow logically from the rule. There were a total of 40 reasoning trials (10 conditional statements, 4 inferences each), all presented in a random order.

In the second part of the experiment, the participants answered interpretation questions about the same statements. They were given extensive explanations about how to answer the necessity and sufficiency questions. The participants were given rephrasings and examples in the instructions and were invited to ask the experimenter if anything was unclear before answering the interpretation questions. Sufficiency questions were phrased as Is the fact that $p$ occurs enough to be sure that $q$ will occur? (e.g., Is the fact that a child eats too much sugar enough to be sure that he will have behavioral difficulties?). Necessity questions were phrased as Does the fact that $q$ occurs guarantee that $p$ has occurred? (e.g., Does the fact that a child has behavioral difficulties guarantee that he or she eats too much sugar?). For each question, participants answered on a scale from 1 (not at all) to 7 (absolutely). The order in which the rules were presented was randomly determined.

\section{Results}

Reasoning task. The average proportions of logically valid answers (MP and MT: yes, DA and AC: no) were entered into a $4 \times 2$ ANOVA, with inference type (MP, DA, AC, MT) and emotionality (emotional, neutral) as within-subjects variables. For all inferential tests, an alpha level of .05 was used throughout this and the other experiments.

The participants were more likely to provide logically valid answers when reasoning about neutral statements $(M=0.72, S D=0.44)$, compared with emotional statements $(M=0.68, S D=0.46)$. This difference was significant $\left[F(1,31)=6.16, M S_{\mathrm{e}}=0.08, \eta_{\mathrm{p}}^{2}=.17\right]$.

The main effect of inference type was also significant $\left[F(3,93)=13.21, M S_{\mathrm{e}}=1.98, \eta_{\mathrm{p}}^{2}=.30\right]$. It can be seen from Figure 1 that the participants provided more logically valid answers in response to MP, followed by AC, MT, and then DA.

The two main effects did not interact $[F(3,93)<1]$. Figure 1 shows that the participants tended to provide more logically valid answers in response to neutral statements for all types of inferences.

Interpretation of conditional statements. Overall, the participants rated the statements as more sufficient $(M=6.28, S D=1.12)$ than necessary $(M=2.28, S D=$ 1.72) $[t(31)=10.54]$, suggesting that they understood the task. The participants' ratings of emotional and neutral statements were compared using paired-samples $t$ tests. There was no difference in sufficiency ratings of emotional $(M=6.21, S D=1.2)$ and neutral rules $(M=6.35$, $S D=1.1)[t(31)=1.38, p=.18]$. The participants' ratings of necessity were also similar for emotional $(M=$ $2.30, S D=1.8)$ and neutral rules $(M=2.25, S D=1.7)$ $[t(31)<1]$. Thus, emotional and neutral statements were not interpreted in significantly different ways.

Relationship between interpretation and reasoning. To examine whether there were links between the way the participants interpreted the statements and their answers on the reasoning task, answers to each of the two interpretation questions (sufficiency, necessity) were entered as predictors in four different standard regression equations, one with each of the four inferences as a dependent variable (MP, DA, AC, MT). Overall, these two independent variables did predict a significant amount of variance in responses to the DA [adjusted $R^{2}=.63, F(2,29)=27.9$ ] and $\mathrm{AC}$ inferences [adjusted $R^{2}=.47, F(2,29)=14.6$ ], but not responses to the MT $[F(2,29)<1]$ or MP inferences $[F(2,29)=1.6, p=.22]$. 


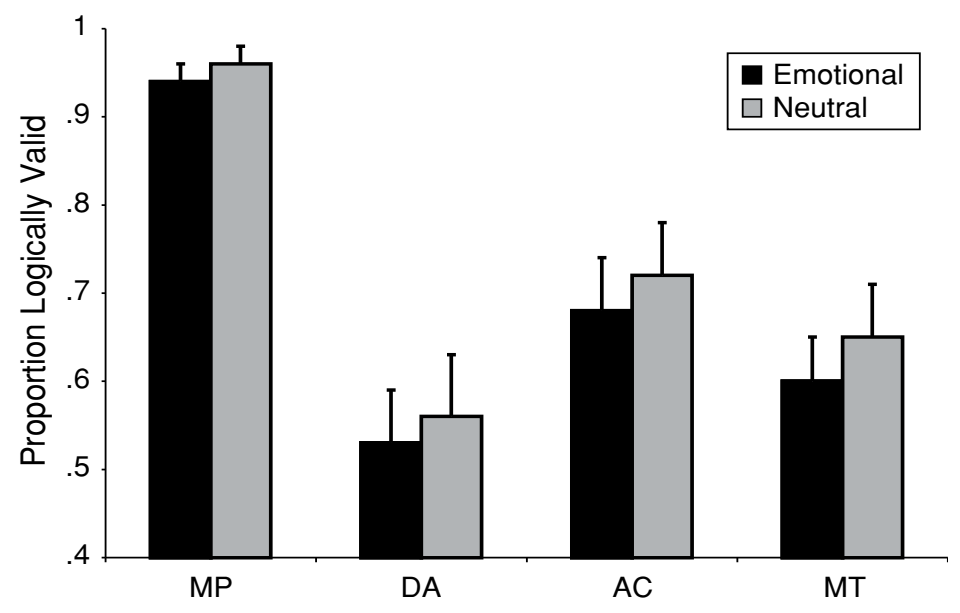

Figure 1. Mean proportions of logically valid answers $(+S E)$ as a function of inference type and emotional connotation, Experiment 1.

Further analyses were carried out to examine whether the relationship between interpretation and endorsement of DA and AC was similar for the two types of statements. Results of the separate equations for emotional and neutral statements are presented in Table 2. Overall, the most important predictor of answers to both DA and AC was perceived necessity. The more participants perceived the relationships to be necessary, the more likely they were to incorrectly endorse DA and AC. Perceived sufficiency was also related to answers on DA and $\mathrm{AC}$, but to a lesser extent. This pattern was similar for emotional and neutral statements.

\section{Discussion}

The goals of Experiment 1A were twofold. The first goal was to test the effect of emotional content on logicality in a conditional reasoning task. The second goal was to examine whether interpretation mediates the effect of emotion on reasoning. The results provided important findings in relation to these two goals.

The participants evaluated the logical validity of conditional inferences for emotional and neutral statements. Answers conformed more closely to the prescriptions of normative logic when the participants reasoned about neutral statements, compared with emotional statements.
Results from this experiment build on previous findings in important ways (Blanchette \& Richards, 2004). In this experiment, there were no ambiguities in the task. The participants were given conclusions and had to determine whether these were logically valid or not. It was emphasized throughout that the participants should rely on logical validity. Thus, this experiment shows that even when a task's requirements for logical reasoning are increased, the effect of emotion remains.

In addition, a new set of materials was employed in this experiment. These were existing statements related to general knowledge. An independent group of participants rated the emotionality of these stimuli. The superficial similarity between the statements used in the emotional and neutral conditions was maximized. Thus, Experiment $1 \mathrm{~A}$ provided a conceptual replication of the effect previously found, not only with a different set of stimuli, but with additional controls.

Notwithstanding these methodological improvements, possible confounds remain. The statements participants reasoned about in the emotional and neutral conditions were different, used different words, and evoked different associations. Although the emotional content was different across conditions, other factors may have varied as well. Using existing emotional and neutral stimuli, it is

Table 2

Summary of Standard Regression Analyses for Interpretive Variables Predicting Responses on the Reasoning Task, Experiment 1A

\begin{tabular}{|c|c|c|c|c|c|c|}
\hline \multirow[b]{3}{*}{ Dependent Variable } & \multirow[b]{3}{*}{ Adjusted $R^{2}$} & \multirow[b]{3}{*}{$F(2,29)$} & \multicolumn{4}{|c|}{ Independent Variables } \\
\hline & & & \multicolumn{2}{|c|}{ Sufficiency } & \multicolumn{2}{|c|}{ Necessity } \\
\hline & & & $\mathrm{B}$ & $\beta$ & $\mathrm{B}$ & $\beta$ \\
\hline \multicolumn{7}{|l|}{ DA } \\
\hline Emotional & .64 & 28.7 & .12 & .41 & -.14 & -.68 \\
\hline Neutral & .50 & 16.4 & .14 & .40 & -.13 & -.57 \\
\hline \multicolumn{7}{|l|}{$\mathrm{AC}$} \\
\hline Emotional & .42 & 12.1 & .05 & .18 & -.12 & -.64 \\
\hline Neutral & .45 & 13.7 & .09 & .31 & -.11 & -.59 \\
\hline
\end{tabular}


impossible to isolate the effect of emotional connotation from other possible associated semantic factors. Furthermore, the influence of perceived sufficiency and necessity may vary depending on the content of the conditional statements used. Although efforts were made to make the statements in the two conditions as superficially similar as possible, it is possible that differences in semantic content muddled both affective and interpretive effects.

To remedy this problem, in Experiment 1B, emotionality of content was manipulated independently from semantic content, using classical conditioning. The methodology involves taking initially neutral words (e.g., sandwich, butter, camera, etc.) and repeatedly pairing them with emotional or neutral images. This pairing is randomly determined for each participant, so that across participants, the same words will be either emotional or neutral. The conditioned words are then included in the If $p$, then $q$ statements that participants reason about. If differences are observed between reasoning about the conditioned emotional and conditioned neutral words, one can unambiguously attribute the effect to the emotional value, and not semantic content of the statements, which is identical in all cases.

\section{EXPERIMENT 1B}

\section{Method}

Participants. The participants were the same as in Experiment 1A.

Materials. Twelve words were used in six conditional statements (see Table 3). These words were neutral, as documented by published ratings of word emotionality (John, 1988). The average emotionality rating of the words was $1.62(S D=0.32)$ on a scale from 1 to 7, where 1 represents not at all emotional. The words were divided into two lists; each would be paired with photographs of neutral or negative emotional valence. The photographs were taken from the International Affective Picture System (Lang, Bradley, $\&$ Cuthbert, 2001). There were 36 photographs of each type. On negativity/positivity scales (1-9), the negative photographs received an average rating of $2.72(S D=0.47)$, compared with 4.93 for the neutral ones $(S D=0.42)$.

Procedure. The participants completed Experiments 1A and 1B together. Experiment 1B included four blocks. The first block was the conditioning paradigm. In the second block, the participants reasoned about conditional statements (those from Experiment 1A first, followed by those for Experiment 1B). In the third block, they answered the necessity and sufficiency questions (again, for the statements from Experiment 1A first, then for Experiment 1B). In the fourth block, the participants rated the emotionality of the words that had been conditioned.
In Block 1, the participants went through the conditioning paradigm. The pairing of the word list and picture type was determined randomly for each participant. The lists included words to be used as $p$ and $q$ in the conditional statements; thus, both would be conditioned with the same type of images. A blocked presentation of the word-picture pairs was used. For instance, all negative conditioning trials were presented first, followed by all neutral trials, or vice versa. This order was randomly determined. Each block involved 100 word-picture pairings. Each word was presented between 16 and 17 times, each time with a different photograph of the same emotional valence.

Each conditioning trial lasted 2,000 msec. The photograph first came up on the computer screen for $500 \mathrm{msec}$. While the picture remained on the screen, the word, written in white on a small black rectangle, then appeared in the middle of the screen for $1,500 \mathrm{msec}$. There was a $500-\mathrm{msec}$ blank screen before the start of the next trial.

In the second block, the participants reasoned about the six conditional statements made up of the conditioned words. In the third block, they answered the necessity and sufficiency questions. The procedure and instructions for the reasoning and interpretation blocks were identical to those used in Experiment 1A. In the fourth block, the participants completed a word-rating task meant to assess the efficiency of the conditioning procedure. The conditioned words were presented one by one on the computer screen in a random order. The instructions given to participants were Your task is simply to indicate whether this word has, to you, a positive, negative, or neutral emotion connotation. They were asked to answer the first thing that came to mind, and to provide responses on a scale from 1 (very negative) to 7 (very positive).

\section{Results}

Reasoning task. The data were analyzed in the same way as in Experiment 1A. Mean proportions of logically valid answers were entered into a $4 \times 2$ ANOVA, with question type (MP, DA, AC, MT) and emotion (negative, neutral) as within-subjects variables. The results are presented in Figure 2.

The participants were less likely to provide logically valid answers when reasoning about negative statements $(M=0.74, S D=0.44)$, compared with neutral statements $(M=0.79, S D=0.42)$. This difference was significant $\left[F(1,31)=10.98, M S_{\mathrm{e}}=0.15, \eta_{\mathrm{p}}^{2}=.26\right]$.

Performance on the different inferences followed the same order as in Experiment 1A. The participants were most accurate in response to MP, followed by AC, MT, and then DA. The effect of question type was significant $\left[F(3,93)=10.15, M S_{\mathrm{e}}=1.52, \eta_{\mathrm{p}}^{2}=.25\right]$.

The two main effects did not interact $[F(3,93)=1.12$, $p=.34]$. As can be seen in Figure 2, the difference be-

Table 3

Reasoning Stimuli Used in Experiments $1 B$ and 2

\begin{tabular}{|c|c|c|}
\hline List 1 & List 2 & List 3 \\
\hline $\begin{array}{l}\text { If someone is an author, } \\
\text { then he creates texts }\end{array}$ & $\begin{array}{l}\text { If something has a nucleus, } \\
\text { then it has a diameter }\end{array}$ & $\begin{array}{l}\text { If something is used as a cloth, } \\
\text { then it contains cotton }\end{array}$ \\
\hline $\begin{array}{l}\text { If someone works as a waitress, } \\
\text { then she receives tips }\end{array}$ & $\begin{array}{l}\text { If someone wears a costume, } \\
\text { then she wears garments }\end{array}$ & $\begin{array}{l}\text { If someone is a businessman, } \\
\text { then he is involved in commerce }\end{array}$ \\
\hline $\begin{array}{l}\text { If something contains dairy, } \\
\text { then it contains proteins }\end{array}$ & $\begin{array}{l}\text { If a person is having pork, } \\
\text { then she is having a meal }\end{array}$ & $\begin{array}{l}\text { If something is going to be in contact with ice, } \\
\text { then it needs to be insulated }\end{array}$ \\
\hline
\end{tabular}




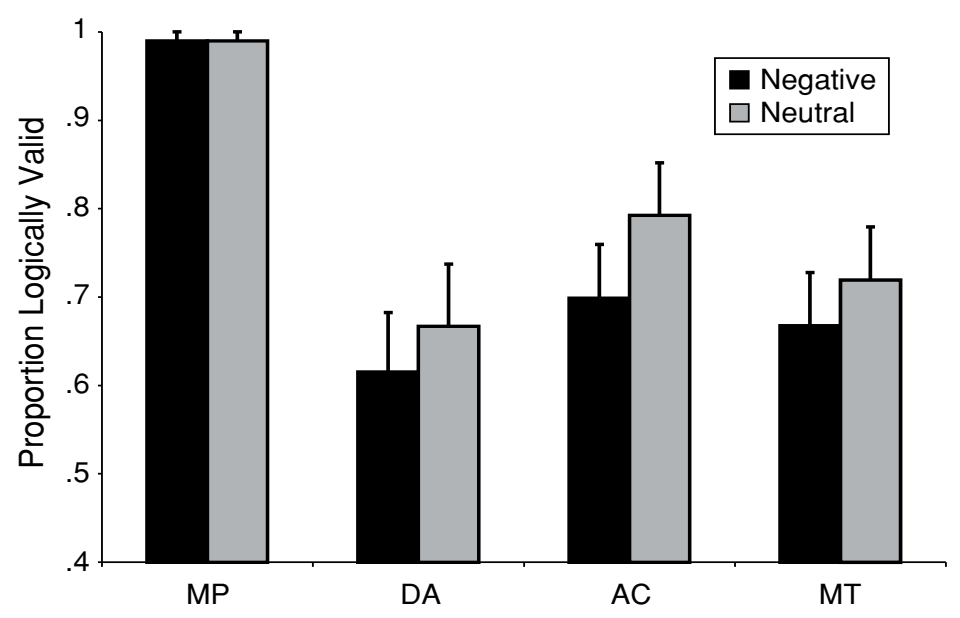

Figure 2. Mean proportions of accurate answers $(+S E)$ for negative and neutral statements, Experiment 1B.

tween negative and neutral statements was in the same direction for all inferences, except for MP, where there was a ceiling effect.

Interpretation of conditional statements. Once again, the participants rated the relationship between $p$ and $q$ as more sufficient $(M=6.59, S D=0.64)$ than necessary $(M=2.23, S D=1.75)[t(31)=13.11]$. Average ratings of sufficiency and necessity for negative and neutral statements were compared using paired-samples $t$ tests. The participants did not rate the sufficiency of negative and neutral statements differently $[M=6.58, S D=$ 0.76 , and $M=6.60, S D=0.75$, respectively; $t(31)<1]$. Furthermore, the participants did not rate the necessity of negative and neutral rules differently $[M=2.34, S D=$ 1.84 , and $M=2.13, S D=1.81$, respectively; $t(31)=$ $1.24, p=.24]$. Thus, negative and neutral statements were not interpreted in significantly different ways.

Relationship between interpretation and reasoning. Regressions were used to examine the relationship between interpretation and responses on the reasoning task. Overall, perceived sufficiency and necessity did contribute to predicting answers to DA [adjusted $R^{2}=.63$,
$F(2,29)=27.6]$ and AC [adjusted $R^{2}=.55, F(2,29)=$ 20.0 ], as well as MP [adjusted $R^{2}=.33, F(2,29)=8.74$ ], but not MT $(F<1)$.

Specific patterns of relation between interpretation and reasoning responses were examined separately for negative and neutral statements. Individual regressions were run separately, with DA, AC, and MP as dependent variables. The results are presented in Table 4. Again, accuracy on DA and AC were strongly, and negatively, related to perception of necessity. The more that participants perceived a relationship to be necessary, the more likely they were to endorse these logical fallacies. Answers to MP were positively related to perceived sufficiency. Again, in all cases, relationships between interpretation and reasoning were comparable for emotional and neutral contents.

Emotionality ratings. The participants rated the negatively conditioned words as more negative $(M=5.15$, $S D=1.00)$ than the neutrally conditioned words $(M=$ $5.59, S D=1.10)$. This difference was significant $[t(31)=$ $2.06]$. Thus, the conditioning procedure did produce the expected effect.

Table 4

Summary of Standard Regression Analyses for Interpretive Variables Predicting Responses on the Reasoning Task, Experiment 1B

\begin{tabular}{|c|c|c|c|c|c|c|}
\hline \multirow[b]{3}{*}{ Dependent Variable } & \multirow[b]{3}{*}{ Adjusted $R^{2}$} & \multirow[b]{3}{*}{$F(2,29)$} & \multicolumn{4}{|c|}{ Independent Variables } \\
\hline & & & \multicolumn{2}{|c|}{ Sufficiency } & \multicolumn{2}{|c|}{ Necessity } \\
\hline & & & B & $\beta$ & B & $\beta$ \\
\hline \multicolumn{7}{|l|}{ DA } \\
\hline Negative & .58 & 22.2 & n.s. & n.s. & -.16 & -.75 \\
\hline Neutral & .44 & 12.9 & n.s. & n.s. & -.15 & -.69 \\
\hline \multicolumn{7}{|l|}{$\mathrm{AC}$} \\
\hline Negative & .51 & 17.2 & n.s. & n.s. & -.14 & -.74 \\
\hline Neutral & .50 & 16.4 & n.s. & n.s. & -.13 & -.73 \\
\hline \multicolumn{7}{|l|}{ MP } \\
\hline Negative & .12 & 3.2 & .03 & .41 & n.s. & n.s. \\
\hline Neutral & .37 & 10.4 & .05 & .64 & n.s. & n.s. \\
\hline
\end{tabular}




\section{Discussion}

Experiment 1B investigated the same issues as Experiment $1 \mathrm{~A}$, but with a methodology that allowed us to unambiguously ascribe any differences between emotional and neutral statements to the actual emotional value of these statements. A conditioning procedure was used to experimentally manipulate the emotional connotation of initially neutral words. Across participants, the same words were conditioned to become more negative or remain neutral. The fact that the same words were for some participants negative and for others neutral allowed us to eliminate any effect of semantic content.

These findings confirm and strengthen those of previous experiments (Blanchette \& Richards, 2004). Again, the participants were less likely to provide normatively correct answers when reasoning about emotional statements. The participants' interpretations of the statements had an important impact on the way they reasoned. Perceived necessity increased the likelihood of endorsing $\mathrm{AC}$ and DA. Perceived sufficiency was related to answers on MP. Together, interpretational factors accounted for a large proportion of the variance in reasoning responses. This suggests that the measures of sufficiency and necessity were sensitive. Furthermore, it is consistent with previous research on the effect of interpretation on reasoning (Thompson, 1994, 1995).

Interpretive factors, however, did not account for the difference in reasoning between emotional and neutral rules. The participants did not perceive emotional rules as less sufficient, or more necessary, which would have been consistent with the differences observed on the reasoning task. In particular, it rules out the possibility that the participants are simply more likely to interpret emotional rules as biconditionals. If the participants indeed interpreted emotional rules as biconditionals, then they should have rated them as more necessary. This was not the case.

The first two experiments explored the role of perceived necessity and sufficiency. These are crucial in determining participants' behavior on conditional reasoning tasks. However, there are other dimensions on which emotional and neutral statements may be interpreted differently. Additional possibilities are explored in Experiment 2.

\section{EXPERIMENT 2}

Previous research has singled out how perceived plausibility of the conditional statement, as well as the strength of the link between $p$ and $q$, affect reasoning (Cummins et al., 1991; De Neys, Schaeken, \& D'Ydewalle, 2002; Markovits, 1986; Politzer \& Bourmaud, 2002; Quinn \& Markovits, 1998). Politzer and Bourmaud (2002) recently reviewed the literature on conditional reasoning involving uncertainty. They provided numerous examples of the fact that the degree of belief in the major premise (or the conditional statement) affects the inferences drawn. In particular, MP and MT are less likely to be endorsed when there is uncertainty about the conditional statement. They suggest that a number of factors, including perceived necessity and disabling conditions, influence reasoning because they af- fect the degree of belief in the major premise. This level of belief (or disbelief) trickles down to inferences drawn based on the major premise and results in certain inferences being more or less likely to be drawn. Thus, one possible interpretational factor that could mediate the effect of emotion on reasoning is plausibility, or the degree of belief in the major premise. The affective connotation of the conditional statement may increase, or decrease, the extent to which people consider it to be plausible, which would, in turn, affect inferences. This is directly tested in Experiment 2 by asking people to rate the plausibility of each statement.

Another related factor is the extent to which $p$ and $q$ are perceived to be strongly, or loosely, associated. De Neys, Schaeken, and D'Ydewalle (2002) have shown that associative strength is related to plausibility, and both factors may affect the likelihood of drawing certain inferences. In addition, associative strength is also related to how many alternative causes can be generated for a conditional statement. The greater the perceived associative strength, the smaller the number of alternative causes that are generated. This might be especially important in determining responses to $\mathrm{DA}$ and $\mathrm{AC}$, where imagining alternative causes can decrease endorsement of the two inferences. Thus, perceived causality represents another factor that could mediate the relationship between emotion and reasoning. If the relationship between $p$ and $q$ is perceived as more causal in the case of emotional stimuli, this could lead to greater endorsement of certain inferences, particularly DA and AC.

In Experiment 2, the crucial feature is again the use of a conditioning procedure. Thus, in the emotional and neutral conditions, the same words are used and semantics are kept constant. This makes it possible to verify whether the affective value of the statement, independently, influences perceived causality and plausibility.

In Experiments 1A and 1B, negative and neutral materials were compared. In previous research, similar effects were found for positive and negative emotional contents on reasoning (Blanchette \& Richards, 2004). Using the conditioning paradigm, it is often more difficult to produce positive changes in affective value than it is to produce negative ones. This may be because participants generally start with a relatively high base rate, or because the stimuli used for negatively conditioning stimuli are generally more powerful. Nevertheless, in Experiment 2, positively conditioned stimuli as well as negatively conditioned stimuli were used to explore potential differences related to valence.

In addition to measuring perceived causality and plausibility, the extent to which the participants were confident in their responses was also measured in Experiment 2. On each trial of the reasoning task, after providing an answer, the participants rated how confident they were in their responses.

\section{Method}

Participants. Twenty-two individuals participated in this study for monetary compensation. The participants were recruited on the campus at the University of Manchester and were mostly students 
or staff of the university. There were 4 men and 18 women in the sample. Mean age was $32.6(S D=11.5)$, ranging from 20 to 54 .

Materials. The same materials used in Experiment 1B were again used, and stimuli necessary for the positive conditioning were added (see Table 3). A total of 18 words were used in nine conditional statements (including the 12 words previously used in Experiment 1B). The words were divided into three lists. Each list would be paired with photographs of neutral, negative, or positive emotional valence. There were again 36 photographs of each type (total number of images $=108)$. As before, the average rating for the negative photographs was $2.72(S D=0.47)$, compared with 4.93 for the neutral ones $(S D=0.42)$. The positive photographs' average rating was $8.06(S D=0.31)$ (Lang et al., 2001).

Procedure. The procedure was similar to that used in Experiment 1B. The participants completed the conditioning block and then the reasoning task, followed by the interpretation block and the manipulation check (affective rating of the conditioned words). One difference was that positively conditioned materials were added to the different blocks.

The participants' confidence in their responses was measured. After each answer, the participants had to indicate on a scale from 1 (essentially guessing) to 7 (absolutely certain) how confident they were in the response they had just provided.

Before the interpretation questions, the participants were given extensive instructions on how to answer the causality and plausibility questions, with reference to examples. The participants were asked to rate the extent to which the first event was causing the second one to occur, on a scale from 1 (absolutely does not cause) to 7 (absolutely does cause). Similarly, they were asked to rate the overall plausibility of the statements on a scale from 1 (absolutely not plausible) to 7 (very plausible).

Another difference with the procedure previously used was in the emotionality ratings (Block 4). In Experiment 1B, the participants rated the words that had been conditioned individually. In the present experiment, the participants rated the rule as a whole, rather than individual conditioned words. The participants again provided answers on a scale from 1 (very negative) to 7 (very positive).

\section{Results}

Reasoning task. Mean proportions of logically valid answers were entered into a $4 \times 3$ ANOVA, with question type (MP, DA, AC, MT) and emotion (negative, neutral, positive) as within-subjects variables. The means are presented in Figure 3.
Emotion had a significant effect on reasoning $\left[F(2,42)=3.94, M S_{\mathrm{e}}=0.13, \eta_{\mathrm{p}}^{2}=.16\right]$. The participants were less likely to provide the logically valid answer when reasoning about negatively conditioned stimuli $(M=.66$, $S D=.20)$ as well as positively conditioned ones $(M=$ $.70, S D=.15)$, compared with neutrally conditioned stimuli $(M=.74, S D=.15)$. The significant quadratic trend associated with the effect of emotion confirms this pattern of differences $[F(1,21)=6.41]$. However, post hoc comparisons using the Bonferroni adjustment procedure revealed that whereas the difference between negative and neutral means reached significance $(p=.04)$, that between neutral and positive means did not $(p=.54)$.

Overall, the participants were most accurate in response to MP, followed by AC, DA, and MT. The main effect of question type was significant $\left[F(3,63)=2.97, M S_{\mathrm{e}}=\right.$ $\left.0.78, \eta_{\mathrm{p}}^{2}=.12\right]$. The effects of emotion and question type did not interact $\left[F(6,126)<1, M S_{\mathrm{e}}=0.003\right]$.

Interpretation of conditional statements. Ratings of causality and plausibility for negative, positive, and neutral statements were compared using separate one-way ANOVAs. Both analyses showed no difference in the ratings as a function of emotion $(F \mathbf{s}<1)$. Negative, neutral, and positive statements were not interpreted differently in terms of causality $(M=4.2, S D=1.4 ; M=4.9, S D=$ $1.2 ; M=4.7, S D=1.5$, respectively) or plausibility $(M=$ 4.7, $S D=1.4 ; M=4.8, S D=1.2 ; M=4.9, S D=1.6$, respectively).

Relationship between interpretation and reasoning. Regressions were used to examine the relationship between interpretation and responses on the reasoning task. Overall, perceived causality and plausibility did contribute to predicting answers to MP [adjusted $R^{2}=.28, F(2,19)=$ 3.64 ] and MT [adjusted $R^{2}=.65, F(2,19)=7.08$ ], but not $\mathrm{AC}$ and DA ( $F \mathrm{~s}=1.7$ and 2.3, respectively).

These relationships were examined separately for negative and neutral statements. Individual regressions were run separately, with MP and MT as dependent variables. The results are presented in Table 5. Generally, the

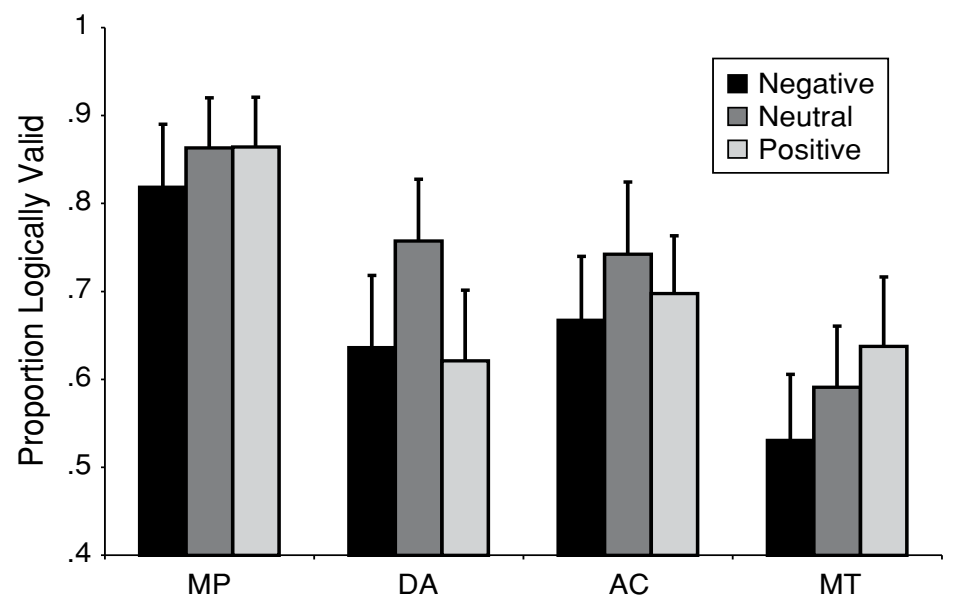

Figure 3. Mean proportions of accurate answers $(+S E)$ for negatively, neutrally, and positively conditioned stimuli, Experiment 2. 
Table 5

Summary of Standard Regression Analyses for Interpretive Variables Predicting Responses on the Reasoning Task, Experiment 2

\begin{tabular}{|c|c|c|c|c|c|c|}
\hline \multirow[b]{3}{*}{ Dependent Variable } & \multirow[b]{3}{*}{ Adjusted $R^{2}$} & \multirow[b]{3}{*}{$F(2,19)$} & \multicolumn{4}{|c|}{ Independent Variables } \\
\hline & & & \multicolumn{2}{|c|}{ Causality } & \multicolumn{2}{|c|}{ Plausibility } \\
\hline & & & $\mathrm{B}$ & $\beta$ & $\mathrm{B}$ & $\beta$ \\
\hline \multicolumn{7}{|l|}{ MP } \\
\hline Negative & .32 & 4.45 & .09 & .44 & n.s. & n.s. \\
\hline Neutral & .29 & 3.89 & .10 & .47 & n.s. & n.s. \\
\hline \multicolumn{7}{|l|}{ MT } \\
\hline Negative & .40 & 7.98 & n.s. & n.s. & .21 & .82 \\
\hline Neutral & .31 & 4.3 & n.s. & n.s. & n.s. & n.s. \\
\hline
\end{tabular}

amount of variance accounted for by the interpretive factors is similar for both types of stimuli. Furthermore, perceived causality seems to be most important in predicting MP inferences, for both negative and neutral statements. For MT, perceived plausibility was most important; however, this did not reach significance in the case of neutral statements.

Confidence ratings. Confidence ratings were compared as a function of emotion (negative, neutral, positive) and accuracy (accurate, inaccurate answers), using a $3 \times 2$ within-subjects ANOVA. Overall, the participants were more confident when they were indeed accurate $(M=5.75, S D=1.06)$ than when they were inaccurate $(M=5.34, S D=1.06)\left[F(1,18)=13.9, M S_{\mathrm{e}}=3.73\right.$, $\left.\eta_{\mathrm{p}}^{2}=.44\right]$. There was no effect of emotion on confidence, and the two main effects did not interact $\left(F_{\mathrm{S}}<1\right)$.

Emotionality ratings. The participants rated the rules with conditioned negative words as the most negative $(M=4.08, S D=0.75)$, followed by statements including neutrally conditioned words $(M=4.50, S D=1.16)$ and statements with positively conditioned words $(M=4.73$, $S D=1.10$ ). A planned linear contrast confirms that the difference between means, in that order, was significant $[F(1,21)=4.6]$.

\section{Discussion}

The effect of emotion on logical reasoning was again replicated in Experiment 2. As in Experiment 1B, emotion was manipulated independently of semantic content. Positive and negative emotional contents produced similar effects on reasoning. Both led to a decrease in logically valid answers, relative to neutral content; however, this did not reach significance in the case of positively conditioned stimuli. This is not unexpected, given previous research. It is generally more difficult to condition stimuli to become more positive than it is to condition them to become more negative. The small sample size in this experiment may have exacerbated this problem.

A new set of interpretive factors was examined in this experiment. The effect of emotion on reasoning, again, was not mediated by these factors. Emotional rules were not perceived as more or less causal or plausible, yet the participants nevertheless reasoned differently as a function of affective content. Perceived causality, as expected, was related to endorsement of MP. The more that partici- pants perceived the relationship between $p$ and $q$ to be causal, the more likely they were to endorse MP, for both negative and neutral rules. MT, in turn, was more strongly related to plausibility, although this only reached significance for negative statements. Again, the small sample size may have prevented the effect from being observed for neutral stimuli.

The conditionals used in these experiments were not designed to present causal relationships. The same materials used in Experiment 1B were used here. The degree to which the participants perceived causality in these preexisting statements was measured, but not manipulated. Another way to test the potential link between causality, reasoning, and emotion would be to compare causal and noncausal conditionals, with emotional and neutral contents. This would also make it possible to test the hypothesis that causality mediates the effect of emotion on reasoning. Furthermore, it is possible that emotion, causality, perceived necessity, and sufficiency actually interact in a complex way, whereby perceived necessity and sufficiency may be stronger determinants of reasoning responses for causal statements that are emotional, compared with those that are not. Given that the two sets of factors were examined in different experiments, it is not possible to rule out this possibility here. The approach taken in Experiment 2 was simply to check whether the same statements (with the same semantic contents) would be interpreted as being more causal, or more plausible, if they were laden with affective connotations. The results showed that this was not the case.

\section{GENERAL DISCUSSION}

The three experiments reported in this article showed that emotion has a systematic effect on conditional reasoning. The participants were less likely to provide normatively correct answers when reasoning about emotional materials, compared with neutral materials. This was true both for existing neutral and emotional stimuli, and for stimuli with experimentally manipulated emotional value. Positive and negative contents produced similar effects. The results also confirmed the importance of interpretive factors on reasoning behavior. Especially in Experiments $1 \mathrm{~A}$ and $1 \mathrm{~B}$, perceived necessity and sufficiency were strongly associated with responses in the reasoning task. 
However, it does not appear that the effect of emotion is mediated by interpretation. Emotional and neutral stimuli were interpreted in similar ways, yet were reasoned about differently. Furthermore, the relationship between interpretation and reasoning was similar for both emotional and neutral materials.

The effect of emotion on logical reasoning in nonclinical samples has up until now been a relatively neglected area of empirical research. Nevertheless, the present results are consistent with previous studies showing effects of different emotion-related constructs (i.e., mood, mood disorders) on logical reasoning. Thus, it appears that both incidental affective states and the emotional value of the contents produce systematic effects on reasoning. This is important, because many topics that people reason about in daily life can be highly emotional (e.g., If a child has been abused, then he may suffer from behavioral difficulties; If you have unprotected sex, you may get sexually transmitted diseases, etc.). In the context of the recent calls for the reasoning literature to address more of the complexities inherent in real-life reasoning (Evans \& Thompson, 2004), emotion seems an important factor to explore.

One important feature of the experiments reported in this article is the increased emphasis on logicality (relative to previous experiments, e.g., Blanchette \& Richards, 2004), both in the type of task that participants completed and in the instructions. A logical validity task was used, and participants were reminded that they must answer on the basis of logical validity on every single trial. Although this did not prevent participants from bringing their background knowledge to bear on the task, it did remove ambiguities in terms of experimental demand, and make it a more stringent test of the effect of emotion on logicality. Thus, even when task demands increase the pressure to use formal reasoning - as opposed to associative, heuristic, or experience-based reasoning - the effect of emotion remains strong.

\section{Emotion, Reasoning, and Interpretation}

Another important feature of these experiments is the exploration of the role of interpretive variables. Recent research has provided several examples of the importance of interpretation in reasoning (Ahn \& Graham, 1999; Liu, 2003; Thompson, 1995, 2000; Thompson \& Mann, 1995). The results from the present experiments confirmed this; especially the roles of perceived necessity and sufficiency. Material implication implies a sufficient but not necessary relation between $p$ and $q$. As expected, increased perception of necessity was particularly related to an increase in the endorsement of logical fallacies (AC and DA). Notwithstanding, emotion did not affect interpretation. If the effect of emotion on reasoning was mediated by interpretation, a specific pattern of interpretation could have been found. The participants tended to endorse AC and DA more in the case of emotional rules. Correspondingly, emotional rules could have been interpreted as implying a more necessary relation between $p$ and $q$. There was no evidence to support this hypothesis.
Clearly, one must be cautious in drawing conclusions based on the absence of effect. In all three experiments, there was no evidence that the participants interpreted emotional statements differently. However, a definite conclusion can never be established based on null effects. Yet, given that the experimental design did allow us to see other important effects of interpretation, the absence of effect in the case of emotion does not stem from an inability to capture the important features and variance of this variable. To put it simply, it was clear that the participants did interpret certain statements differently (as more causal, less sufficient, etc.) and that this was directly related to their reasoning. It was also clear that they perceived different statements as more or less emotionally laden. Although the results showed both of these effects, they did not show a link between interpretation and emotion. If the effect of emotion on reasoning was entirely mediated by these interpretive factors, we should have seen an effect. In other words, if interpretation were the main mechanism through which emotion affects reasoning, it would have produced differences in the way the stimuli were interpreted. Because no such differences were observed, it can at least be concluded that interpretation is not the only mechanism involved, and maybe not the most important one. Nevertheless, it cannot be concluded here that emotion does not have any effect on interpretation.

A more general issue, however, relates to what is considered an interpretive effect. In this article, I have used the term interpretation in a rather strict sense, to refer to the dimensions known to be important in determining people's behavior on logical reasoning tasks. This could be termed the "logical interpretation" of the stimuli. Interpretation can be construed more generally to include all aspects that affect encoding of stimuli. For instance, it is known that emotion increases the accessibility of stimuli of the same valence in working memory. It could be argued that - especially using the conditioning procedure, when the conditional statements are encoded during the reasoning task - representations of the emotional images previously presented are primed, and this affects reasoning behavior. This would relate to encoding, and could be said to relate to interpretation in the broader sense. The conclusions from the experiments conducted here are only relevant to the more strict definition of interpretation.

Considering strict logical interpretation, results from these experiments are consistent with the idea that the effect of emotion may be on "reasoning proper," or the inferential aspect of the task. If this is the case, there are several ways in which this could occur. Different models of conditional reasoning exist, and each suggests different possibilities in terms of mechanisms.

\section{Models of Reasoning and the Effect of Emotion}

Probabilistic models have recently been proposed to account for the behavior of participants on conditional reasoning tasks. The basic idea is that people reason probabilistically about If $p$, then $q$ statements, and essentially answer logical reasoning tasks based on the perceived probability of the different co-occurrence of $p$, not $p, q$, 
and not $q$. For instance, there is evidence showing that the evaluation of the truth of If $p$, then $q$ statements is directly related to the frequency of the occurrence of the different options. Generally, the more likely it is that $q$ occurs in the presence of $p$, and the less likely it is to occur in the absence of $p$, the more the conditional will be judged to be true, and this increases endorsement of logically valid inferences. There may be good reasons why emotions affect perceived utility and probability. Emotional events are thought to be, by definition, important to one's current goals. Emotional reactions are not elicited by trivial events; in fact, there is some evidence consistent with the hypothesis that perceived utility of negative and positive events in the context of conditional statements may vary. For instance, in the research of de Jong and colleagues looking at danger and safety rules (de Jong et al., 1998; de Jong et al., 1997; Smeets et al., 2000), $q$ and not $q$ are clearly of different subjective utility, depending on the type of rule. These experiments have varied semantic or informational content simultaneously with emotional value. Nevertheless, it is possible that emotional value could independently affect perception of utility and probability. There is also some evidence that emotion influences covariation estimates, especially in the case of fear (Pauli, Wiedemann, \& Montoya, 1998; Pury \& Mineka, 1997). Thus, one possibility is that emotion influences reasoning through affecting perceived probabilities and subjective utility of the different options. This could be construed as leading back to an interpretive account of the effect of emotion on reasoning. Subjective utility and probability are related to the way conditionals are interpreted. I would argue that these are related to interpretation in the more general sense, and not the strict logical sense. This is consistent with the fact that probabilistic models represent an alternative to formal deductive reasoning models.

Mental models and mental logic are two classes of models that, although quite different in many aspects, share important features. In both cases, it is assumed that an initial representation is built (spatially or propositionally), and that systematic processes of inferences are then applied to that representation (Braine \& O'Brien, 1998; Johnson-Laird, 1983; Johnson-Laird \& Byrne, 1991). Thus, in both cases, there would need to be a mechanism that can act within the inferential component and implement the effect of emotion. Such a mechanism could be working memory (WM). Both classes of models ascribe an important functional role to working memory, particularly in constraining the number of computations that can be performed. For instance, it is thought that WM limitations are responsible for the fact that participants do not represent exhaustively all possibilities of a conditional statement, which leads them to endorse logically invalid inferences. There is ample evidence that variations in WM capacity are indeed related to reasoning performance, both within (Gilhooly, Logie, Wetherick, \& Wynn, 1993) and across participants (Stanovich, 1999). There is also some evidence, and some speculation, that mood affects WM capacity. In their study of conditional reasoning using the Wason selection task, Oaksford and colleagues
(1996) observed that the effect of positive mood, at least, seemed to be mediated by reduced WM capacity. Other emotions, such as anxiety, have been shown to be associated with reduced WM span (Derakshan \& Eysenck, 1998; MacLeod \& Donnellan, 1993). However, other studies have shown that positive and negative moods may differentially affect specific components of working memory (Gray, 2001; Perlstein, Elbert, \& Stenger, 2002). There is no corresponding demonstration, as far as I am aware, that processing emotional materials also taxes WM capacity. In fact, one recent article has documented a pervasive lack of effect across a number of tasks (Kensinger \& Corkin, 2003). Nevertheless, speculatively speaking, this is a possible mechanism through which emotion may affect reasoning. Emotional contents may prime a number of associations that load working memory. When using the conditioning procedure, memory traces of the emotional images - more memorable - may be activated to a greater extent than memory traces of neutral images. This mechanism would be consistent with both mental models and mental logic approaches to conditional reasoning.

From the perspective of the reasoning literature, judgments on the rationality of emotion effects on reasoning may depend on the theoretical interpretation adopted. Probabilistic views of conditional reasoning suggest that participants, by relying on probabilities and perceived utility, are actually producing quite rational patterns of responses, even if these do not correspond to the prescriptions of normative logic. It is argued that for reasoning in complex environments, in which knowledge is essentially probabilistic, these mechanisms represent the best possible adaptation. There may be good reasons why emotions affect perceived utility and probability. An increase in the perceived utility of emotional outcomes, for instance, which would lead to differences in reasoning patterns, would be perfectly rational. Mental models or mental logic theories may cast the role of emotion as one of those factors ultimately determining bounded rationality. In these systems, although the inferential aspects could, in theory, unfold perfectly logically, they are limited by the capacity of WM. This often prevents reasoners from reaching the perfectly logical solution, yet WM restrictions ultimately serve other purposes that increase adaptability. Thus, in both types of accounts, the negative influence of emotion on logicality does not necessarily undermine rationality. More generally, the question of the effect of emotion on logicality can be tackled empirically, in a relatively focused way. The experiments reported here provide some answers on this issue. This does not, however, settle the wider issue of rationality.

One last note to put the results of this investigation in perspective. These studies examined the effect of incidental emotional value. Especially in Experiments 1B and 2, the emotional value was dissociated from the semantic content of the problems. This was necessary to isolate the influence of purely affective factors. There are situations in real life in which people reason about issues that have an emotional value, but this aspect is irrelevant to their task. There are, however, other situations in which people 
reason about issues that are affectively charged, but the emotional value is actually a very important feature of the problem. For instance, in the example given previously, If a child has been abused, then he may suffer from behavioral difficulties, the emotional value associated with this problem may signal that the consequences of reasoning about this statement are very important, and as such provide important information to the reasoner. Both incidental and integral effects of emotion are important to study. However, it should not be assumed that results obtained in one line of inquiry generalize to the other.

\section{REFERENCES}

Ahn, W.-K., \& Graham, L. M. (1999). The impact of necessity and sufficiency in the Wason four-card selection task. Psychological Science, 10, 237-242.

Barrett, L. F., \& Salovey, P. (2002). The wisdom in feeling: Psychological processes in emotional intelligence. New York: Guilford.

Blanchette, I., \& Richards, A. (2004). Reasoning about emotional and neutral materials: Is logic affected by emotion? Psychological Science, 15, 745-752.

Braine, M. D. S., \& O’Brien, D. P. (Eds.) (1998). Mental logic. Mahwah, NJ: Erlbaum.

ChAnnon, S., \& BAKER, J. (1994). Reasoning strategies in depression: Effects of depressed mood on a syllogism task. Personality \& Individual Differences, 17, 707-711.

Chater, N., \& OAKsford, M. (2001). Human rationality and the psychology of reasoning: Where do we go from here? British Journal of Psychology, 92, 193-216.

Cheng, P. W., \& Holyoak, K. J. (1985). Pragmatic reasoning schemas. Cognitive Psychology, 17, 391-416.

Cummins, D. D., Lubart, T., Alksnis, O., \& Rist, R. (1991). Conditional reasoning and causation. Memory \& Cognition, 19, 274-282.

Damasio, A. R. (1998). Emotion and reason in the future of human life. In B. Cartledge (Ed.), Mind, brain, and the environment: The Linacre Lectures 1995-6 (pp. 57-71). New York: Oxford University Press.

DAWES, R. M. (2001). Everyday irrationality: How pseudo-scientists, lunatics, and the rest of us systematically fail to think rationally. Boulder, CO: Westview Press.

de Jong, P. J., Haenen, M.-A., Schmidt, A., \& Mayer, B. (1998). Hypochondriasis: The role of fear-confirming reasoning. Behaviour Research \& Therapy, 36, 65-74.

De Jong, P. J., Mayer, B., \& van den Hout, M. (1997). Conditional reasoning and phobic fear: Evidence for a fear-confirming reasoning pattern. Behaviour Research \& Therapy, 35, 507-516.

De Neys, W., Schaeken, W., \& D'Ydewalle, G. (2002). Causal conditional reasoning and semantic memory retrieval: A test of the semantic memory framework. Memory \& Cognition, 30, 908-920.

Derakshan, N., \& Eysenck, M. W. (1998). Working memory capacity in high trait-anxious and repressor groups. Cognition \& Emotion, 12, 697-713.

DE SousA, R. (1987). The rationality of emotion. Cambridge, MA: MIT Press.

Ekman, P., \& Davidson, R. J. (EDS.) (1994). The nature of emotion: Fundamental questions. New York: Oxford University Press.

Evans, J. St. B. T. (1989). Bias in human reasoning: Causes and consequences. Hillsdale, NJ: Erlbaum.

Evans, J. ST. B. T. (1998). Matching bias in conditional reasoning: Do we understand it after 25 years? Thinking \& Reasoning, 4, 45-82.

Evans, J. St. B. T., Over, D. E., \& MankTelow, K. I. (1993). Reasoning, decision making and rationality. Cognition, 49, 165-187.

Evans, J. St. B. T., \& Thompson, V. A. (2004). Informal reasoning: Theory and method. Canadian Journal of Experimental Psychology, 58, 69-74.

Fairley, N., Manktelow, K., \& Over, D. (1999). Necessity, sufficiency, and perspective effects in causal conditional reasoning. Quar- terly Journal of Experimental Psychology: Human Experimental Psychology, 52A, 771-790.

French, P. A., \& Wettstein, H. K. (Eds.) (1998). Philosophy of emotions. Notre Dame, IN: University of Notre Dame Press.

Gilhooly, K. J., Logie, R. H., Wetherick, N. E., \& Wynn, V. (1993). Working memory and strategies in syllogistic-reasoning tasks. Memory \& Cognition, 21, 115-124.

Goel, V., \& Dolan, R. J. (2003). Explaining modulation of reasoning by belief. Cognition, 87, B11-B22.

GraY, J. R. (2001). Emotional modulation of cognitive control: Approach-withdrawal states double-dissociate spatial from verbal two-back task performance. Journal of Experimental Psychology: General, 130, 436-452.

Henle, M. (1962). On the relation between logic and thinking. Psychological Review, 69, 366-378.

JoHn, C. H. (1988). Emotionality ratings and free-association norms of 240 emotional and non-emotional words. Cognition \& Emotion, 2, 49-70.

JOHNSON-LAIRD, P. N. (1983). Mental models: Towards a cognitive science of language, inference, and consciousness. Cambridge, MA: Harvard University Press.

Johnson-Laird, P. N., \& Byrne, R. M. J. (1991). Deduction. Hillsdale, NJ: Erlbaum.

Kensinger, E. A., \& Corkin, S. (2003). Effect of negative emotional content on working memory and long-term memory. Emotion, 3, 378393.

Klaczynski, P. A., \& Narasimham, G. (1998). Representations as mediators of adolescent deductive reasoning. Developmental Psychology, 34, 865-881.

LANG, P. J., BRADley, M. M., \& CUTHBert, B. N. (2001). International Affective Picture System (IAPS): Instruction manual and affective ratings (Tech. Rep. A-5). Gainesville, FL: University of Florida, The Center for Research in Psychophysiology.

LEFFORD, A. (1946). The influence of emotional subject matter on logical reasoning. Journal of General Psychology, 34, 127-151.

LIU, I.-M. (2003). Conditional reasoning and conditionalization. Journal of Experimental Psychology: Learning, Memory, \& Cognition, 29, 694-709.

LyONS, W. (1993). Emotion. Aldershot, U.K.: Gregg Revivals.

MacLeod, C., \& Donnellan, A. M. (1993). Individual differences in anxiety and the restriction of working memory capacity. Personality \& Individual Differences, 15, 163-173.

MankTelow, K. (1999). Reasoning and thinking. Hove, U.K.: Psychology Press.

Markovits, H. (1986). Familiarity effects in conditional reasoning. Journal of Educational Psychology, 78, 492-494.

Markovits, H., \& Nantel, G. (1989). The belief-bias effect in the production and evaluation of logical conclusions. Memory \& Cognition, 17, 11-17.

Melton, R. J. (1995). The role of positive affect in syllogism performance. Personality \& Social Psychology Bulletin, 21, 788-794.

OAKSFORD, M., \& CHATER, N. (1998). Rationality in an uncertain world: Essays on the cognitive science of human reasoning. Hove, U.K.: Psychology Press.

OAKSFORD, M., Morris, F., Grainger, B., \& Williams, J. M. G. (1996). Mood, reasoning, and central executive processes. Journal of Experimental Psychology: Learning, Memory, \& Cognition, 22, 476-492.

Oнm, E., \& Thompson, V. A. (2004). Everyday reasoning with inducements and advice. Thinking \& Reasoning, 10, 241-272.

Parrott, W. G. (1995). But emotions are sometimes irrational. Psychological Inquiry, 6, 230-232.

Pauli, P., Wiedemann, G., \& Montoya, P. (1998). Covariation bias in flight phobics. Journal of Anxiety Disorders, 12, 555-565.

Perlstein, W. M., Elbert, T., \& Stenger, V. A. (2002). Dissociation in human prefrontal cortex of affective influences on working memoryrelated activity. Proceedings of the National Academy of Sciences, 99, 1736-1741.

Politzer, G., \& Bourmaud, G. (2002). Deductive reasoning from uncertain conditionals. British Journal of Psychology, 93, 345-381.

Pury, C. L. S., \& MineKA, S. (1997). Covariation bias for blood-injury 
stimuli and aversive outcomes. Behaviour Research \& Therapy, $\mathbf{3 5}$ $35-47$.

Quinn, S., \& Markovits, H. (1998). Conditional reasoning, causality, and the structure of semantic memory: Strength of association as a predictive factor for content effects. Cognition, 68, B93-B101.

Radenhausen, R. A., \& Anker, J. M. (1988). Effects of depressed mood induction on reasoning performance. Perceptual \& Motor Skills, 66, 855-860.

Smeets, G., DE Jong, P. J., \& Mayer, B. (2000). If you suffer from a headache, then you have a brain tumor: Domain-specific reasoning "bias" and hypochondriasis. Behaviour Research \& Therapy, 38, 763-776.

Stanovich, K. E. (1999). Who is rational? Studies of individual differences in reasoning. Mahwah, $\mathrm{NJ}$ : Erlbaum.

THOMPson, V. A. (1994). Interpretational factors in conditional reasoning. Memory \& Cognition, 22, 742-758.

Thompson, V. A. (1995). Conditional reasoning: The necessary and sufficient conditions. Canadian Journal of Experimental Psychology, 49, $1-60$.
Thompson, V. A. (2000). The task-specific nature of domain-general reasoning. Cognition, 76, 209-268.

Thompson, V. A., \& ManN, J. M. (1995). Perceived necessity explains the dissociation between logic and meaning: The case of "Only If." Journal of Experimental Psychology: Learning, Memory, \& Cognition, 21, 1554-1567.

Verbrugge, S., Dieussaert, K., Schaeken, W., \& Van Belle, W. (2004). Promise is debt, threat another matter: The effect of credibility on the interpretation of conditional promises and threats. Canadian Journal of Experimental Psychology, 58, 106-112.

Verschueren, N., Schroyens, W., Schaeken, W., \& D'Y dewalle, G. (2001). Why do participants draw non-valid inferences in conditional reasoning? Current Psychology Letters: Behaviour, Brain \& Cognition, 6, 57-70.

(Manuscript received February 23, 2005; revision accepted for publication June 16, 2005.) 